\title{
Rice Bran Oil: Applications Food Products, Characteristics Physicochemical, Extractions, and Purifications (A Review)
}

\author{
Vira Putri Yarlina ${ }^{1}$, Edy Subroto $^{2}$, Aldila Din Pangawikan ${ }^{3}$, Mohammad Djali $^{4}$, Robi Andoyo $^{5}$, Risya Aulia $^{6}$ \\ ${ }^{1}$ Universitas Padjadjaran, Indonesia, vira.putri.yarlina@ unpad.ac.id \\ ${ }^{2}$ Universitas Padjadjaran, Indonesia, edy.subroto@unpad.ac.id \\ ${ }^{3}$ Universitas Padjadjaran, Indonesia, pangawikan@unpad.ac.id \\ ${ }^{4}$ Universitas Padjadjaran, Indonesia, djali@unpad.ac.id \\ ${ }^{5}$ Universitas Padjadjaran, Indonesia, r.andoyo@unpad.ac.id \\ ${ }^{6}$ Universitas Padjadjaran, Indonesia, risyaaulia@unpad.ac.id
}

\begin{abstract}
Rice bran oil is a vegetable oil that has specific characteristics and has health benefits. The rice bran oil phytochemical components such as tocopherols, tocotrienols, squalene, phytosterols, polyphenols, and $\gamma$ oryzanol. There are component rarely found in other vegetable oils. Rice bran oil has good oxidation stability which is very beneficial in deep-frying pans. This review aims to determine characteristics of rice bran oil such ass physicochemical properties, the extractions and purifications, and its application in food products. Rice bran oil has health benefits and quality, namely antioxidant, anti-cancer, and cardiovascular disease. Rice bran oils can be used for spreadable bran oil, margarine and various food products.
\end{abstract}

Key words: Rice Bran oil, physicochemical, extraction, purification, application food products

\section{INTRODUCTION}

Indonesia is one of largest country that consumed and processed Oryza sativa into rice. Before consumption, Oryza sativa must be cleaned from the skin to produce rice bran. In the process of milling,Oryza sativa produced $57-60 \%$ rice yield, $18-20 \%$ husk, and $8-10 \%$ bran. Bran is one of a byproduct rice milling process, consisting of an outer layer of rice grains with a number of seeds. Rice bran contains protein (13.11-17.19\%), Fat (2.52-5.05\%), Carbohydrate (375,91-387,2 calories). Rice bran not only have main component, but also contains phenolic, vitamins, and minerals [1].

The extraction of bran oil from fat contents of rice bran. The process of extraction rice bran, such as stabilization and solid-liquid extraction. The aims of extraction and stabilization to destroy lipase enzyme using heat and chemical to produced and increased yield of rice bran oil[1]. The physical characteristics of the rice bran oil are bright color, medium taste, long- shelf life, and high smoke point. Therefore, rice bran oil is a popular in several countries and continues to be developed [2].

Rice bran oil contains $85 \%$ triacylglycerol, oil that one of the proportions of ingredients [3]. Rice bran oil have bioactive nutrients and antioxidants, that are tocopherols, tocotrienols, squalene, phytosterols, polyphenols, and $\gamma$ oryzanol. Rice bran oil commonly used to cooking and premium edible oil, especially in Asian Countries. Rice bran oil (RBO) is known as Functional food or Health Food. It because, rice bran oil has bioactive nutrients can be used to health benefit. RBO have benefits to anticancer, cardiovascular disease, and cholesterol disease.

The recent application of rice bran oil such as spreaderablerice bran oil and margarine [4]. The journal review aims to provide information about rice bran oil that can be developed especially in the food sector and determine physicochemical, extractions, purifications of rice bran oil.

\section{PHYSICO-CHEMICALCHARACTERISTICS}

\section{a. Physical Characteristics}

Purified rice bran oil has a color value in $5 \mathrm{R}+\mathrm{Y}$ units. The oil for rice bran that is purified by chemical means is 13.8-15.5, while by physical means it is 7.6-13.9. Rice bran oil has darker color oil than sunflower oil, kesumba oil, and peanut oil [5], [6]. The color of the rice bran oil will change with the length of heating into reddish brown. The change in color turns dark due to the thermal 
oxidation process and the phospholipids that darken on heating [7].

Crude rice bran oil has $1.5-4 \%$ wax content, $0.5-1.5 \%$ phosphatides, and $59.19 \%$ free fatty acids. The moisture of rice bran oil is $28.46 \%$, the humidity will increase during repeated frying. The viscosity value is $3.39 \times 10$ $6 \mathrm{~m}^{2} \cdot \mathrm{s}^{-1}$ which will increase due to heating during frying. Increasing the viscosity of rice bran oil produces a layer bond between fried foods and frying oil [8]. Total polar formation increases with repeated frying cycles and is associated with changes in viscosity in the oil [9].

\section{b. Chemical characteristics}

Unsaturated fatty acids content of rice bran oil is quite high, such as $45 \%$ oleic acid and $33 \%$ linoleic acid. Rice bran oil contains phytochemical components such as tocopherols, tocotrienols, squalene, phytosterols, polyphenols, and $\gamma$-oryzanol [10]. The bioactive components from rice bran oil showeda physiological activity as antioxidants, anti-inflammatory and hyperlipidemic [11].

The rice bran contains high acid due to the water content in ethanol which causes the rice bran oil to be hydrolyzed during the extraction process. Rice bran oil has non-polar properties so that rice bran oil can dissolve using nonpolar solvents [12]. Bran oil consists of oleic acid, linoleic acid, linolenic acid, palmitic acid, stearic acid, and ara chidonic acid (Afinisha Deepam \& Arumughan, 2012; Kaneto et al., 2010). The free fatty acids in bran oil are higher than other vegetable oils, about $70 \%$ consisting of oleic acid and linoleic acid, and 22\% palmitic acid [6], [13]-[15].

Rice bran oil has oxidative stability better than palm oil, its strong stability of antioxidant during heating process. The antioxidants in rice bran oil break down oxidation by adding hydrogen atoms to free radicals. Rice bran oil is considered to have oxidative stability equal to even better than soybean oil, corn canola oil, kesumba oil in the use of deep frying [16].

\section{THE EXTRACTIONS AND PURIFICATIONS}

The extraction of rice bran includes the solvent method and the press method. In the press method generally uses cold pressing and hot pressing. Extraction of rice bran oil using a solvent method which generally uses hexane as a solvent. This method will produce high free fatty acids. This is because the extraction method uses high temperatures so free fatty acids are formed in the bran oil. High free fatty acids can be reduced by $\mathrm{NaOH}$ at the oil refining stage in the neutralization process. However, the concentration of $\mathrm{NaOH}$ in oil refining needs to be considered, because the high concentration of $\mathrm{NaOH}$ can remove around $90 \%$ of the antioxidant gamma oryzanol in rice bran oil [17], [18]. The extraction method using hexane is not beneficial in foods so this method is rarely used [19].

Pressing method especially cold pressing is generally used to produce large scale rice bran oil. This method is considered easier, environmentally friendly because it does not require a lot of energy. This method suitable for small or medium scale industries. The difference between cold and hot-pressing methods is the use of the temperature used. The hot press method uses a screw pressing machine with a temperature of $80-100{ }^{\circ} \mathrm{C}$ [20].

The extraction of rice bran oil has 2 process, such as stabilization as a pre-treatment and extraction by solidliquid extraction. The aim of stabilization is to protect rice bran oil from degradation during storage. The degradation of triglycerides in the oil causes the present of off-flavors and unpleasant odors from rice bran oil due to storage of oil for a long time at room temperature [15].

Stabilization methods include heating methods (microwave heating and evaporative ohmic heating), cooling and $\mathrm{pH}$ reduction. The heating of the rice bran is formed from the change in mechanical energy to heat from the screw [20]. Heating the bran at high temperature will reduce the humidity in the bran thereby reducing the need for drying.

Bran stabilization was carried out by placing the bran in a microwave-save polyethylene package and then heating it for 3 minutes at a temperature of $120^{\circ} \mathrm{C}$. Next process, brancolled at room temperature overnight and repeated three times until it reaches the appropriate stabilization [19]. High temperatures above $120^{\circ} \mathrm{C}$ will denature the enzymes so degradation of lipids without damaging nutrients in the bran. The stabilized bran is then cooled in a cooling tray for 30 minutes so that it reaches room temperature.

Rice bran oil produced without stabilization will produce less oil extract. Rice bran without stabilization also has a higher humidity than stabilization. After stabilization, the bran is more brittle so that during treatment it is easier to reach the tissue and increases oil extraction during mechanical pressing [20]. The rice bran is ready to be extracted to obtain coarse rice bran oil after stabilization process. 
Purification of rice bran oil aim to reach of nutritional composition with health benefits. The wax and free fatty acids such as monoacylglycerol and diacylglycerol still have in rice bran oil [21]. Purification process by remove unnecessary substances in oil. Purification consists of degumming, neutralization, bleaching, dewaxing, and deodorizing process[8].

Pigments for rice bran include chlorophyll, lutein, xanthopyl, caratenoids and protein. This pigment is easily degraded in the process so that it is stated as brown pigment. In addition, impure oil contains other impurities that are harmful to health such as soap residue, phospholipids, metal ions, and residual pesticides. To reduce these things, a bleaching process is needed. The bleaching process in bran oil is different from other vegetable oils and tends to be difficult due to the content of chlorophyll, red pigment, oxidation of tocopherol, and free acid metal salts in the oil [22].

The bleaching process aims to remove unwanted compounds, including changing the color of the oil to increase the rate of rancid reactions that come from triglycerides and produce oxidation-forming reaction. The bleaching processes in rice bran oil is the adsorption process using activated bone charcoal [22]. The results of bleaching using activated bone charcoal are able to absorb impurities in rice bran oil. The greater the active surface area of the absorption, the more impurities will be absorbed so that the oil is purer. The blanched rice bran oil still quite high of water content is above $0.2 \%$ by weight of oil, so it needs to be removed in the further deodorizing process.

The refined oil has changes in physicochemical parameters and antioxidant activity. Deodorization process causes a decrease in tocopherol content due to oxidation reactions or chemical reactions such as the formation of tocopherol esters degradation at high temperature by $21.71 \%$ [23], [24]. The neutralization process of rice bran oil causes a high loss of gamma oryzanol, phytosterols, polyphenols and antioxidant activity [17].

\section{THE APPLICATIONS OF RICE BRAN OIL IN FOOD PRODUCTS}

Rice bran oil along with the development of technology is increasingly being developed. Not only as cooking oil, rice bran oil but also developed as a food ingredient to support products that have better benefits and quality. The applications of rice bran oil that have been developed in food products shows in Table 1.

\section{a. Rice bran oil produce spreadable bran products}

Over the past two decades allergies to peanuts or peanut plants have increased. The high severity of allergy makes sufferers have to avoid these ingredients completely. In America, peanut products are one of the most popular products. Therefore, there is a need for other alternatives for people with allergies to peanuts.

The process of spreadable bran oil products is derived from rice bran oil which has a high of waxes. Spreadable rice bran oil produced by mixed rice bran oil with acetone at two different temperatures. This aims to investigate the effects of temperature and time on the chemical composition. The texture result of the spreadable rice bran product resembles butternut [3].

The main components of spread bran oil are TAG and wax in the oil. Samples with a faster incubation time contain higher levels of bran while the TAG is lower. Longer incubations have a higher proportion of bran and TAG. Longer incubation time in spread sample resulted in better C-18 fatty acids [4].

The product of crude rice bran oil spread has 40-70\% concentrations of tocopherols, tocotrienols, and oryzanols. The concentration increases with longer sample incubation. D-tocopherol is a component that does not depend on the length of the incubation time. Phytosterols are found in spread products compared to crude bran oil. The sterols of crude rice bran oil have a concentration of $109-321 \%$ and this concentration 10-12x higher in spread products [4], [13].

\section{b. Spreads of rice bran oil as a functional nutritions}

Rice bran oil spread products have been successfully incorporated into baking products with good reception. Earlier baking products commercially used hydrogenated oil to achieve their desired attributes. The use of semisolid fractionated rice bran oil is free of fat and allergens. The antioxidant properties, free of trans fats, and allergens make the product a semi-solid and structured lipid suitable for development as a functional components [4].

The tocopherols and tocotrienols in spread products are lower than rice bran oil, this is because the spread product has very low melted milk but is still in an adequate range. Tocopherols and tocotrienols highly levels contribute the activity of antioxidants. Spreadable with faster incubation times have higher elastic dodulity compared to longer 
incubations [26]. Myristic acid, palmitic acid, and wax in the bran were higher at the faster incubation time. This is because the component is solid at room temperature, causing a stiffer spread [4].

Table 1: The Applications of Rice Bran Oil in Food Products

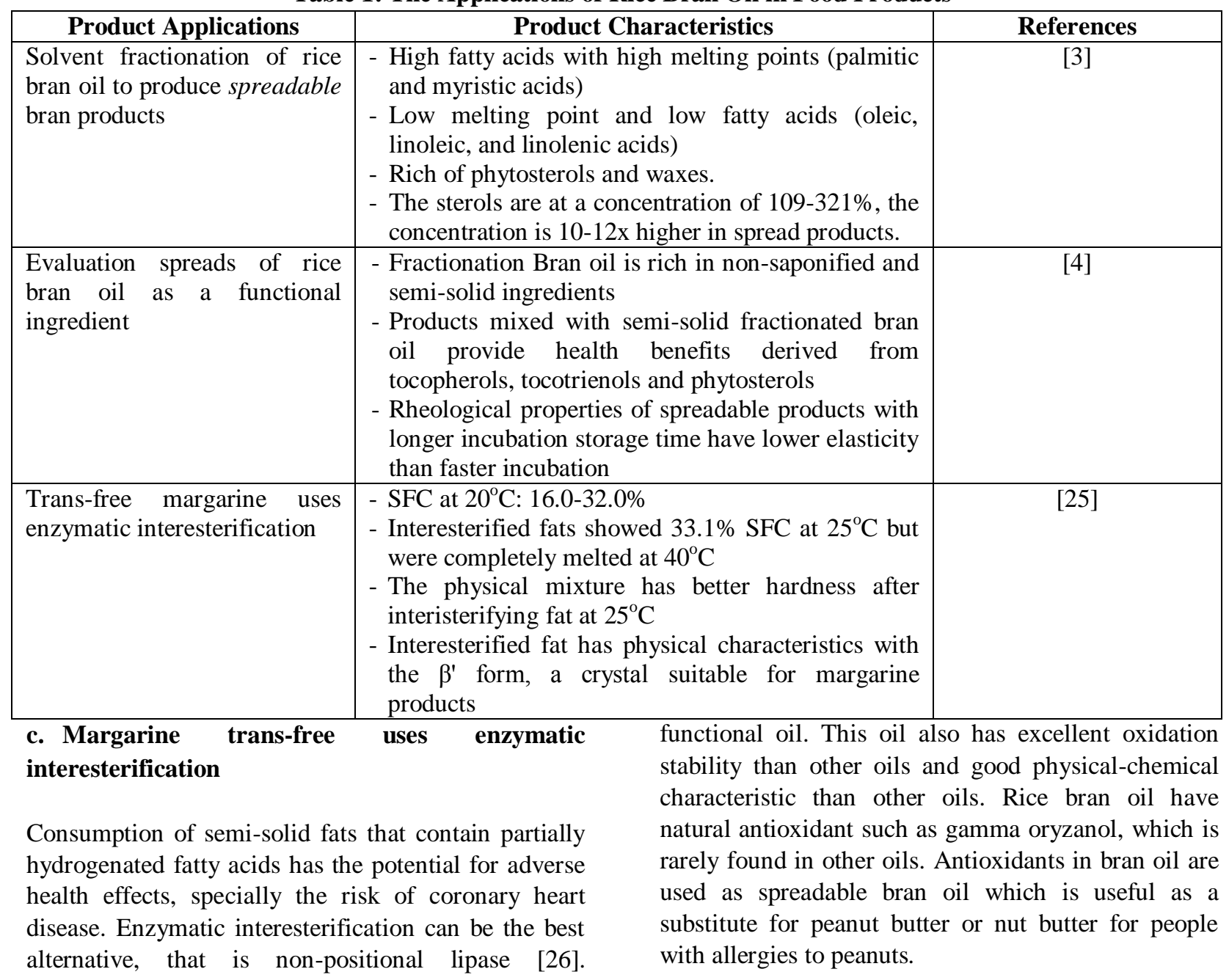

Triacyglycerols Medium chain (TRM) contain longchain saturated fatty acids of 6-12 carbon atoms. This TRM is very stable to oxidation due to its saturation. TRM is also hydrolyzed more rapidly and completely than long chain thiacylglycerols (TRP). Palm stearin functions as the desired hardness forming as margarine and coconut oil aim to improve nutritional aspects, because of high medium-chain fatty acid content. The interesterified fat has the desired characteristics, which is to have a suitable melting point and a crystalline shape (ex: more $\beta$ 'crystals) so it is possible as margarine products[26].

\section{CONCLUSION}

Rice bran oil is one of a vegetable oils derived from extract fatfromrice bran. The bioactive components of rice bran oil are benefit for health. In addition, rice bran oil is widely developed in food products as a

\section{CONFLICT OF INTEREST}

All authors no conflict of interest for this journal

\section{ACKNOWLEDGMENT}

Thanks for all support provided from Universitas Padjadjaran and the Ministry of Education and Culture of the Republic of Indonesia.

\section{REFERENCES}

1. S. Handayani, S. Fajirah, and E. Enjarlis, Rice Bran Oil Extraction as Trioxolane Raw Material,Simetrikal J. Eng. Technol., vol. 2, no. 1, pp. 13-19, 2020, doi: 10.32734/jet.v2i1.3063.

2. G. Ahmad Nayik, I. Majid, A. Gull, and K. Muzaffar, Rice bran oil, the Future Edible Oil 
of India: A mini Review, Rice Res. Open Access, vol. 03, no. 04, 2015, doi: 10.4172/23754338.1000151.

3. E. L. Bakota, J. K. Winkler-Moser, H. S. Hwang, M. J. Bowman, D. E. Palmquist, and S. X. Liu, Solvent fractionation of rice bran oil to produce a spreadable rice bran product, Eur. J. Lipid Sci. Technol., vol. 115, no. 8, pp. 847857, 2013, doi: 10.1002/ejlt.201200355.

4. E. L. Bakota, J. K. Winkler-Moser, and S. X. Liu, Evaluation of a rice bran oil-derived spread as a functional ingredient,Eur. J. Lipid Sci. Technol., vol. 116, no. 5, pp. 521-531, 2014, doi: 10.1002/ejlt.201300259.

5. G. Sasipriya and P. Siddhuraju, Effect of different processing methods on antioxidant activity of underutilized legumes, Entada scandens seed kernel and Canavalia gladiata seeds, Food Chem. Toxicol., vol. 50, no. 8, pp. 2864-2872, 2012, doi: 10.1016/j.fct.2012.05.048.

6. A. G. Gopala Krishna, K. H. Hemakumar, and S. Khatoon, Study on the composition of rice bran oil and its higher free fatty acids value, JAOCS, J. Am. Oil Chem. Soc., vol. 83, no. 2, pp. 117-120, 2006, doi: 10.1007/s11746-006-11831.

7. R. B. Latha and D. R. Nasirullah, Physicochemical changes in rice bran oil during heating at frying temperature, J. Food Sci. Technol., vol. 51, no. 2, pp. 335-340, 2014, doi: 10.1007/s13197-011-0495-9.

8. V. R. Pestana-Bauer, R. C. Zambiazi, C. R. B. Mendonça, M. Beneito-Cambra, and G. RamisRamos, $\boldsymbol{\gamma}$-Oryzanol and tocopherol contents in residues of rice bran oil refining, Food Chem., vol. 134, no. 3, pp. 1479-1483, 2012, doi: 10.1016/j.foodchem.2012.03.059.

9. S. Debnath, N. K. Rastogi, A. G. Gopala Krishna, and B. R. Lokesh, Effect of frying cycles on physical, chemical and heat transfer quality of rice bran oil during deep-fat frying of poori: An Indian traditional fried food, Food Bioprod. Process., vol. 90, no. 2, pp. 249256, 2012, doi: 10.1016/j.fbp.2011.05.001.

10. S. N. Patel, M., \& Naik, Gamma-oryzanol from rice bran oil - A review,J. Sci. Ind. Res. (India)., vol. 63, no. 7, pp. 569-578, 2004.

11. Y. P. C. Rao, D. Sugasini, and B. R. Lokesh, Dietary gamma oryzanol plays a significant role in the anti-inflammatory activity of rice bran oil by decreasing pro-inflammatory mediators secreted by peritoneal macrophages of rats, Biochem. Biophys. Res. Commun., vol. 479, no. 4, pp. 747-752, 2016, doi: 10.1016/j.bbrc.2016.09.140.

12. C. Dorni, P. Sharma, G. Saikia, and T. Longvah,
Fatty acid profile of edible oils and fats consumed in India, Food Chem., vol. 238, no. May, pp. 9-15, 2018, doi: 10.1016/j.foodchem.2017.05.072.

13. L. S. Afinisha Deepam and C. Arumughan, Effect of saponifi cation on composition of unsaponifi able matter in rice bran oil, J. Oleo Sci., vol. 61, no. 5, pp. 241-247, 2012, doi: 10.5650/jos.61.241.

14. H. Kaneto, N. Katakami, M. Matsuhisa, and T. A. Matsuoka, Role of reactive oxygen species in the progression of type 2 diabetes and atherosclerosis, Mediators Inflamm., vol. 2010, 2010, doi: 10.1155/2010/453892.

15. K. Srikaeo and M. Pradit, Simple techniques to increase the production yield and enhance the quality of organic rice bran oils, J. Oleo Sci., vol. 60, no. 1, pp. 1-5, 2011, doi: 10.5650/jos.60.1.

16. S. R. Valantina, V. Mukesh Kumar, and T. Devasena, Selected rheological characteristics and physicochemical properties of vegetable oil affected by heating, Int. J. Food Prop., vol. 19, no. 8, pp. 1852-1862, 2015, doi: 10.1080/10942912.2015.1024849.

17. R. Liu et al., Effect of refining process on physicochemical parameters, chemical compositions and in vitro antioxidant activities of rice bran oil, $L w t$, vol. 109, pp. 2632, 2019, doi: 10.1016/j.lwt.2019.03.096.

18. V. Van Hoed, J. V. Ayala, M. Czarnowska, W. De Greyt, and R. Verhé, Optimization of physical refining to produce rice bran oil with light color and high oryzanol content, JAOCS, J. Am. Oil Chem. Soc., vol. 87, no. 10, pp. 12271234, 2010, doi: 10.1007/s11746-010-1606-x.

19. M. K. Chatha, S. A. S., Hussain, A. I., Zubair, M., \& Khosa, Analytical characterization of rice (oryza sativa) bran and bran oil from different agro-ecological regions,Pakistan $J$. Agric. Sci., vol. 48, no. 3, pp. 243-249, 2011.

20. A. Thanonkaew, S. Wongyai, D. J. McClements, and E. A. Decker, Effect of stabilization of rice bran by domestic heating on mechanical extraction yield, quality, and antioxidant properties of cold-pressed rice bran oil (Oryza saltiva L.),LWT - Food Sci. Technol., vol. 48, no. 2, pp. 231-236, 2012, doi: 10.1016/j.lwt.2012.03.018.

21. B. K. De and J. D. Patel, Effect of different degumming processes and some nontraditional neutralizing agent on refining of RBO, J. Oleo Sci., vol. 59, no. 3, pp. 121125, 2010, doi: 10.5650/jos.59.121.

22. D. M. Suhartono, J., Noersalim, C., Mustari, P. L., \& Olivia, The Effect of Stirring Speed on Rice Bran Oil Bleaching Through Adsorption 
Process Using Activated Bone Charcoal,Pros. Semin. Nas. Tek. Kim. “'Kejuangan,” 2011, doi: B01-1-B01-6.

23. C. Tang, G. Tao, Y. Wang, Y. Liu, and J. Li, Identification of $\alpha$-Tocopherol and Its Oxidation Products by Ultra-Performance Liquid Chromatography Coupled with Quadrupole Time-of-Flight Mass Spectrometry, J. Agric. Food Chem., vol. 68, no. 2, pp. 669-677, 2020, doi: 10.1021/acs.jafc.9b06544.

24. A. Verleyen, T., Kamal-Eldin, A., Dobarganes, C., Verhe, R., Dewettinck, K., \& Huyghebaert, Modeling of $\alpha$-tocopherol loss and oxidation products formed during thermoxidation in triolein and tripalmitin mixtures, Lipids, vol.
36, no. 7, pp. 719-726, 2001, doi: https://doi.org/10.1007/s11745-001-0777-6.

25. K. T. Adhikari, P., Shin, J. A., Lee, J. H., Hu, J. N., Zhu, X. M., Akoh, C. C., \& Lee, Production of trans-free margarine stock by enzymatic interesterification of rice bran oil, palm stearin and coconut oil, J. Sci. Food Agric., vol. 90, no. 4, pp. 703-711, 2010, doi: https://doi.org/10.1002/jsfa.3872.

26. P. Adhikari et al., Production of trans-free margarine stock by enzymatic interesterification of rice bran oil, palm stearin and coconut oil, J. Sci. Food Agric., vol. 90, no. 4, pp. 703-711, 2010, doi: 10.1002/jsfa.3872. 